\title{
The added value of multidetector CT in the diagnosis of gastrointestinal causes of acute abdomen in geriatrics
}

\author{
Doaa M. Emara* ${ }^{*}$, Adel M. Rizk, Abeer M. Ahmed and Amr Magdi El Abd
}

\begin{abstract}
Background: Due to changes in the gastrointestinal system in geriatric people, there are higher rates of atypical presentations of common disorders. In order to decrease the mortality rate, an efficient and correct diagnosis should be given for these patients. The aim of this study was to assess the role of MDCT in evaluating gastrointestinal tract-related acute abdominal pain in geriatric patients.

Results: Among fifty geriatric patients presented clinically with acute abdominal pain of gastrointestinal origin from November 2019 to September 2020, mean age was $70.2 \pm 5.6$ years ranging from 65 to 86 years. Majority were female, (thirty patients, 60\%). Gastric causes (16\%): obstruction (62.5\%) and perforation (37.5\%); and intestinal causes (84\%): obstruction (71.4\%), inflammatory (28.6\%), perforation (23.8\%), vascular (11.9\%), and others (2.4\%) were the frequent causes of acute abdominal pain in the studied patients. MDCT had a very high sensitivity of 98\% (95\% Cl) as well as its PPV $(95 \% \mathrm{Cl})$ was $100 \%$ in the evaluation of gastrointestinal-related acute abdominal pain.

Conclusion: MDCT is a reliable diagnostic imaging modality for geriatric patients presented with acute abdominal pain suggested to be of gastrointestinal origin with a very high sensitivity in diagnosing the causative pathological conditions. MDCT can efficiently differentiate between obstructive, inflammatory, perforated, and ischemic bowel disorders.
\end{abstract}

Keywords: Multidetector computed tomography (MDCT), Geriatric patients, The elderly, Acute abdominal pain, Gastrointestinal, Intestinal

\section{Background}

Acute abdominal pain is defined as pain of less than 1 week in duration. It is the most common emergency department (ED) complaint and the fourth most common complaint among geriatric patients. As the mean age of the population is increasing, the number of emergency department visits for the geriatric population is also increasing [1-3].

Although there have been improvements in the management of geriatric patients in the emergency department, these geriatric patients with acute abdominal pain remain to be a diagnostic challenge for ED physicians because of influenced history taking due to cognitive impairment, diminished immune function, as well as many

* Correspondence: dr.emara_doaa@yahoo.com

Department of Radiodiagnosis, Faculty of Medicine, University of Alexandria, Alexandria, Egypt

\section{Springer Open}

diseases which evolve differently in geriatric patients. Furthermore, geriatric patients have a diminished sensorium which is allowing pathology to advance to a very dangerous state before developing symptoms [1, 4-6]. Hence, diagnostic accuracy is lower, and mortality is much higher in this age group than in younger patients. Early and correct diagnosis for the geriatric patients complaining of acute abdomen is critical and significantly affects the outcome of these patients [3].

The causes of abdominal pain are extremely diverse. Gastrointestinal (GI) diseases are common in older patients, and the clinical presentation, complications, and treatment may be different from those in younger patients. With the marked increase in population of aged 65 years and over, the study and care of GI disorders should be a high priority for both clinicians and researchers. Both the

(c) The Author(s). 2021 Open Access This article is licensed under a Creative Commons Attribution 4.0 International License, which permits use, sharing, adaptation, distribution and reproduction in any medium or format, as long as you give appropriate credit to the original author(s) and the source, provide a link to the Creative Commons licence, and indicate if changes were made. The images or other third party material in this article are included in the article's Creative Commons licence, unless indicated otherwise in a credit line to the material. If material is not included in the article's Creative Commons licence and your intended use is not permitted by statutory regulation or exceeds the permitted use, you will need to obtain permission directly from the copyright holder. To view a copy of this licence, visit http://creativecommons.org/licenses/by/4.0/. 
usual course of aging and the accumulation of multiple disease states can lead to impairments in GI function [6].

Imaging modalities play an increasingly important role in the diagnostic workup and management of geriatric patients with acute abdominal pain, with conventional radiography, ultrasonography, and computed tomography all being used in this situation [1, 6]. Ultrasonography is available in most emergency departments and could be used to screen patients or assess specific symptoms [1], as it is inexpensive and free from contrast or ionizing radiation. It is the initial imaging of choice for biliary tract diseases $[2,6]$.

In the care of geriatric patients, $\mathrm{CT}$ is accurate for diagnosing the cause of acute abdominal pain, particularly when it is of gastrointestinal surgical origin [6]. MDCT has many advantages as better contrast bolus exploitation, isotropic imaging [7], and the shortened acquisition time decreases motion artifacts [8].

The aim of this study was to assess the role of multidetector computed tomography (MDCT) in evaluating gastrointestinal tract-related acute abdominal pain in geriatric patients and document the sensitivity of MDCT and the incidence of the different gastrointestinal pathologies presenting as acute abdomen with characterization of each pathology.

\section{Methods}

In a prospective study, 50 geriatric patients who presented with referral criteria of acute abdominal pain that was clinically suspected to be of gastrointestinal origin and were evaluated with multidetector computed tomography (MDCT) between November 2019 and September 2020 were enrolled in this study. Twenty five of the studied patients underwent erect abdominal X-ray, and 20 out of them had abdominal ultrasound preceding the implementation of MDCT. The final diagnosis was confirmed by surgical findings in 29 patients, histopathological examination in 13 patients, and clinical response to medical treatment in 8 patients.

Inclusion criteria are as follow:

- Patients with clinically acute abdominal pain and suspected to be of gastrointestinal origin aging 65 years or older

- Patients with chronic abdominal pain were eligible if there had been an acute change in pain during the previous 7 days

Exclusion criteria are as follow:

- People diagnosed with extra-gastrointestinal cause of abdominal pain like pain of renal origin

- Patients with acute abdominal pain aging less than 65 years old

\section{Protocol for MDCT}

MDCT was performed using 64 slice/rotation Philips CT machines. Non-enhanced CT (NECT) abdomen was done from the level of diaphragm to the symphysis pubis within a single breath hold. The scanning parameters used were $\mathrm{kVp} 120-140$, mAs $150-450$, tube rotation time $0.33 \mathrm{~s} /$ rotation, slice thickness $0.5-1 \mathrm{~mm}$, collimation $6 \mathrm{~mm}$, and reconstruction interval $1 \mathrm{~mm}$.

IV contrast was injected in 39 patients with normal creatinine clearance result, non-ionic iodinated contrast material namely (Iopromide $300 \mathrm{mg} / \mathrm{mL}$ ) was injected through the antecubital vein. The total amount of injected contrast material was injected according to the body weight of the patient $(1.5 \mathrm{~mL} / \mathrm{kg})$. Post contrast images were obtained-during arterial phase starting at $15-30 \mathrm{~s}$ and portal venous phase at $70 \mathrm{~s}$ after the contrast administration. Finally, delayed scans were done starting $5 \mathrm{~min}$ from the starting point. IV contrast was not injected in 11 patients with low creatinine clearance result. Oral positive contrast medium (diluted iodinated water-soluble contrast material) was administrated in two patients of suspected perforation. Water enema was used in 10 cases with suspected large bowel abnormities.

\section{Data analysis and interpretation}

The MDCT images were reconstructed using filtered reconstruction algorithms. The thin slices were sent to the workstation (IntelliSpace Portal Philips workstation) after reconstruction of the raw data using medium soft tissue reconstruction algorithms. Images were interpreted by using multi-planner reconstruction (axial, sagittal, and coronal) views, MIP (maximum intensity projection), and curved MPR (multi-planner reformatted images).

Two radiologists with 21 and 17 years of experience in abdominal imaging interpreted the imaging findings in consensus.

Data were coded and entered using the Statistical Package for the Social Sciences (SPSS) version 25 (IBM Corp., Armonk, NY, USA). Data were summarized and described using the mean as a measure of central tendency, standard deviation as a measure of dispersion for quantitative variables, and frequencies (number of cases) and relative frequencies (percentages) for categorical variables.

Diagnostic analysis was used to assess the role of MDCT in the evaluation of gastrointestinal-related acute abdominal pain in geriatric patients.

\section{Results}

The current study was conducted upon fifty geriatric patients who presented clinically with acute abdominal pain of gastrointestinal origin, mean age was $70.2 \pm 5.6$ years ranging from 65 to 86 years, as $70 \%$ of the patients lie between (65-70 years), while the least age group was from ( $86-90$ years) which constituted only $2 \%$. Majority 
Table 1 Distribution of the studied patients according to age and gender $(n=50)$

\begin{tabular}{|c|c|c|c|c|c|c|}
\hline \multirow{3}{*}{$\begin{array}{l}\text { Age } \\
\text { groups }\end{array}$} & \multicolumn{4}{|c|}{ Gender } & \multirow{2}{*}{\multicolumn{2}{|c|}{ Total }} \\
\hline & \multicolumn{2}{|c|}{ Male } & \multicolumn{2}{|c|}{ Female } & & \\
\hline & No & $\%$ & No & $\%$ & No & $\%$ \\
\hline $65-70$ & 13 & 26 & 22 & 44 & 35 & 70 \\
\hline $71-75$ & 4 & 8 & 3 & 6 & 7 & 14 \\
\hline $76-80$ & 1 & 2 & 4 & 8 & 5 & 10 \\
\hline $81-85$ & 2 & 4 & 0 & 0 & 2 & 4 \\
\hline $86-90$ & 0 & 0 & 1 & 2 & 1 & 2 \\
\hline Total & 20 & 40 & 30 & 60 & 50 & 100 \\
\hline
\end{tabular}

were females (thirty patients, 60\%), while males constituted $40 \%$ of the study sample (Table 1 ).

\section{Distribution of the patients according to MDCT diagnosis (gastric Vs intestinal causes)}

In the current study, acute abdominal pain due to gastric causes was detected in eight patients (16\%) while in forty-two patients (84\%) was due to intestinal causes. Out of the gastric causes, gastric obstruction was found in five patients (62.5\%)-three mesenteroaxial gastric volvulus (Fig. 1) and two gastric outlet obstruction. Gastric perforation was found in three patients (37.5\%)-two perforated peptic ulcer disease and one perforated gastric carcinoma (Fig. 2) (Table 2).

Out of the intestinal causes in patients, thirty $(71.4 \%)$ were due to obstruction, twelve (28.6\%) were due to inflammatory causes, ten $(23.8 \%)$ were due to perforation, five (11.9\%) were due to vascular causes, and only one with colo-cholecystic fistula (Table 3).

\section{Intestinal obstructive causes}

Small intestinal obstruction was detected in twenty patients (66.7\%) (Fig. 3), while large bowel obstruction was detected in ten patients (33.3\%) (Fig. 4). Mechanical bowel obstruction was detected in twenty-six patients (86.7\%), while functional bowel obstruction was detected in four patients (13.3\%). Intestinal obstruction due to neoplastic causes (Figs. 5 and 6) was detected in seven patients (23.3\%), while twenty-three patients (76.7\%) were due to non-neoplastic causes (Figs. 7, 8, and 9).

\section{Intestinal inflammatory causes}

Intestinal inflammatory causes were detected in twelve patients (28.6\%)-acute diverticulitis frequently was detected in five patients $(41.7 \%)$, acute appendicitis was detected in four patients (33.3\%), one patient (8.3\%) had acute non-complicated diverticulitis associated with neoplastic lesion and acute non-complicated appendicitis (Fig. 10), Crohn's disease was detected in one patient $(8.3 \%)$, and one patient $(8.3 \%)$ had stercoral colitis (Fig. 11). Distribution of patients according to intestinal inflammatory causes as regards occurrence of complications or not are presented in Table 4 and Figs. 12 and 13.

\section{Intestinal perforation}

Intestinal perforation was seen in ten patients (23.8\%); eight (80\%) were due to inflammatory causes (seen in acute

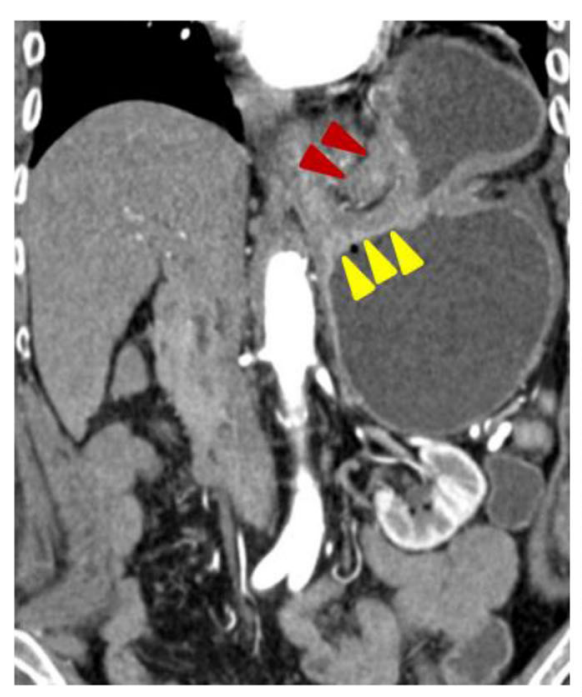

(A)

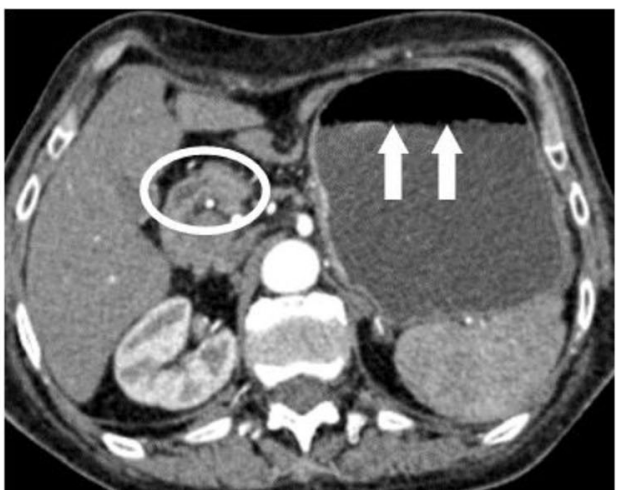

(B)

Fig. 1 Mesenteroaxial gastric volvulus in a 70-year-old female patient presented with sudden onset of vomiting and epigastric pain. a Coronal reformatted contrast-enhanced $\mathrm{CT}$ image shows gastric obstruction secondary to mesenteroaxial volvulus with the gastric antrum (red arrowheads) located superior to the gastroesophageal junction (yellow arrowheads). b Axial contrast-enhanced CT image shows gastric distention with air-fluid levels (arrows) with decompressed duodenum (circle) 


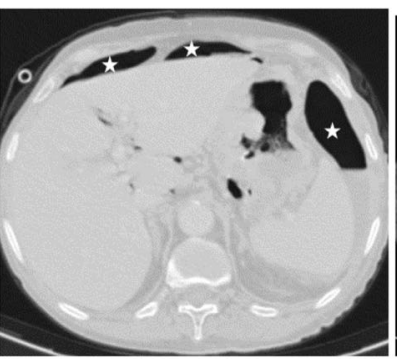

(A)

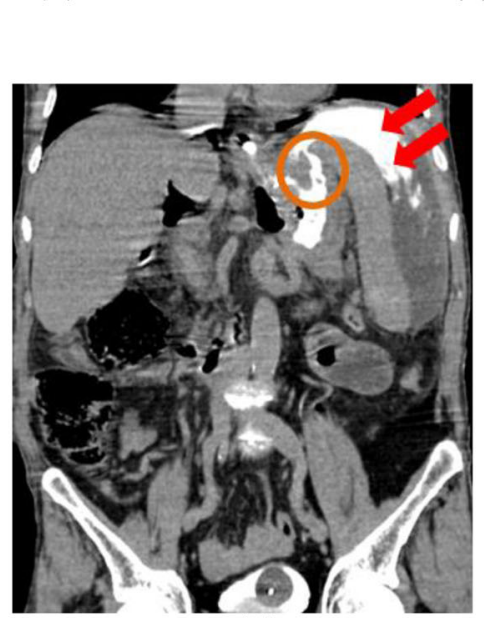

(D)

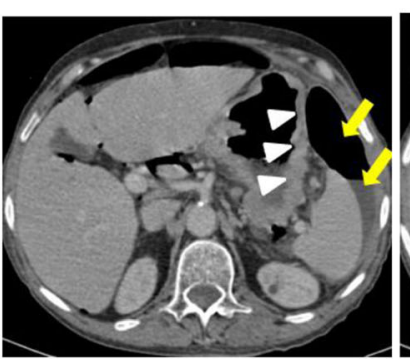

(B)

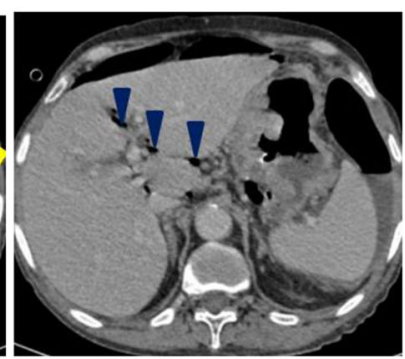

(C)

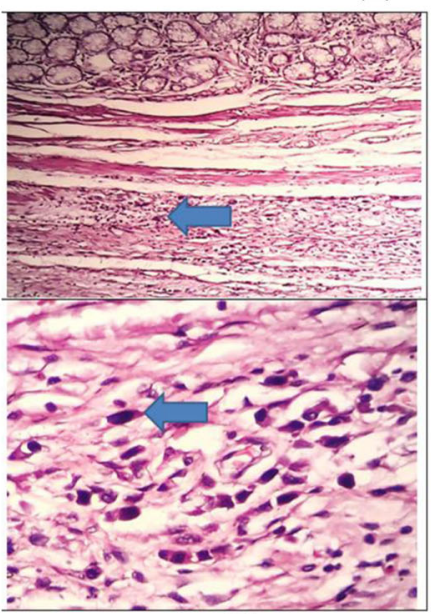

(E)

Fig. 2 Perforated gastric carcinoma in a 73-year-old male patient presented with acute epigastric pain. a Non-contrast CT axial (lung window) image, showing a supramesocolic pneumoperitoneum (stars). b Post IV contrast CT axial image shows diffuse enhancing irregular thickening of gastric wall more evident at the fundal region (white arrowheads) and hydro-pneumoperitoneum (yellow arrows). c Post IV contrast CT axial image showing free air in ligamentum teres (blue arrowheads). $\mathbf{d}$ CT coronal post IV and oral contrast CT image, showing active contrast leakage on delayed phase (red arrows) through a focal defect in posterior gastric fundal wall (circle). e Histopathology showing infiltration of the deep muscle layer by sheets of highly pleomorphic tumor cells, confirming radiological findings of gastric carcinoma

diverticulitis, acute appendicitis, and active Crohn's disease) and two (20\%) were due to neoplastic causes (Fig. 14.)

\section{Intestinal vascular causes}

Vascular causes were detected in five patients (11.9\%). Four patients had acute bowel ischemia due to mesenteric vascular occlusion (two patients (40\%) had superior mesenteric artery (SMA) occlusion; one was complete and the other one was partial occlusion \& two patients $(40 \%)$ had complete superior mesenteric vein (SMV)

Table 2 Distribution of patients according to gastric causes

\begin{tabular}{lll}
\hline Gastric causes & Frequency (No.) & Percent (\%) \\
\hline Gastric obstruction & $\mathbf{5}$ & $\mathbf{6 2 . 5}$ \\
Mesenteroaxial gastric volvulus & 3 & 37.5 \\
Gastric outlet obstruction & 2 & 25 \\
Gastric perforation & $\mathbf{3}$ & $\mathbf{3 7 . 5}$ \\
Perforated PUD & 2 & 25 \\
Perforated gastric carcinoma & 1 & 12.5 \\
Total & $\mathbf{8}$ & $\mathbf{1 0 0}$ \\
\hline
\end{tabular}

occlusion (Fig. 15)), as well only one patient (20\%) had acute small bowel wall hematoma (Fig. 16).

\section{Colo-cholecystic fistula}

Colo-cholecystic fistula was detected only in one patient (2.4\%) (Fig. 17).

\section{Different MDCT findings among the studied patients}

Bowel wall enhancement patterns

Majority of the patients had optimal enhancement (53.8\%) followed by heterogeneous enhancement pattern

Table 3 Distribution of patients according to intestinal causes

\begin{tabular}{lll}
\hline Intestinal causes & Frequency (No.) & Percent (\%) \\
\hline Obstruction & 30 & 71.4 \\
Inflammatory & 12 & 28.6 \\
Perforation & 10 & 23.8 \\
Vascular & 5 & 11.9 \\
Colo-cholecystic fistula & 1 & 2.4
\end{tabular}

Some patients have two causes at the same time 


\section{Small Bowel Obstruction}

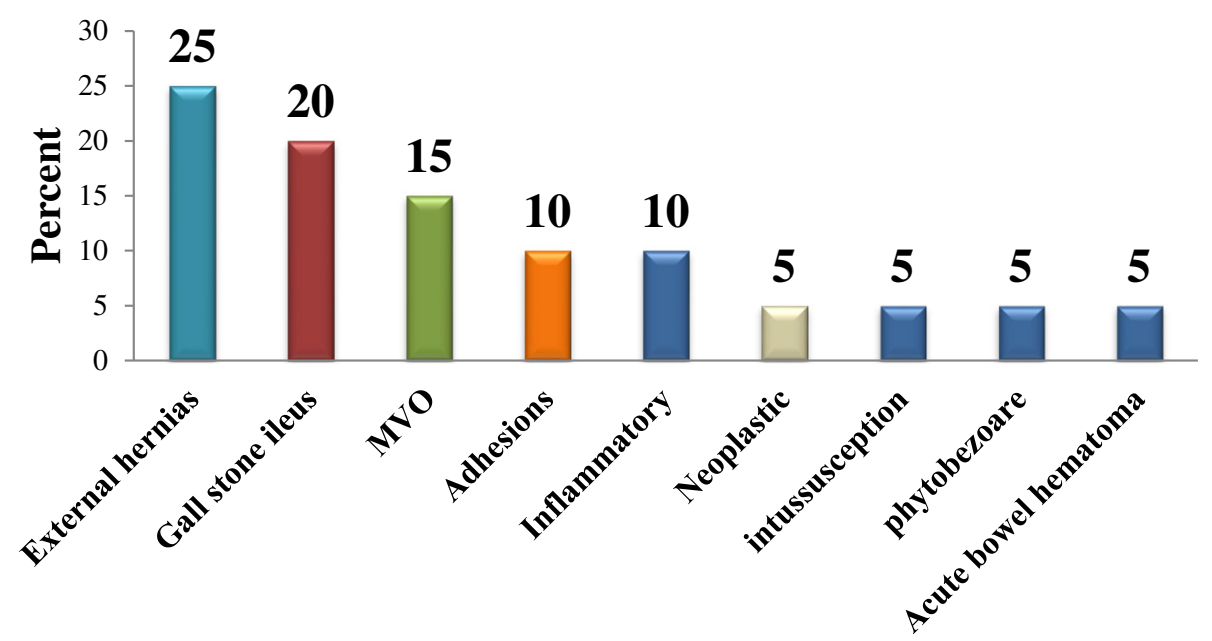

Fig. 3 Distribution of patients according to the causes of small bowel obstruction

(20.5\%). Hyper-enhancement was detected in acute inflammatory conditions (15.3\%), one of them showing target pattern in active Crohn's disease. Non-enhancement was detected in acute irreversible mesenteric ischemia (7.6\%) and only one case showing hypo-enhancement in reversible mesenteric ischemia.

\section{Bowel mural thickening patterns}

This was encountered in nineteen patients (38\%). Twelve patients had focal thickening (63.2\%); ten cases were malignant and two cases were inflammatory, while segmental thickening was seen in seven patients $(36.8 \%)$ in benign conditions. Eleven patients had symmetrical thickening (57.9\%) in benign conditions. Eight patients had asymmetrical thickening (42.1\%) detected in malignant lesions. Four patients had bowel mural thickening in the form of a target sign appearance (21.1\%) seen in Crohn's disease, venous MVO, intussusception, and acute bowel hematoma.

\section{Bowel loop dilatation}

This was detected in thirty patients $(60 \%)$ : twenty patients had small bowel loop dilatation (66.7\%) due to small bowel obstruction and ten patients (33.3\%) had large bowel loop dilatation due to large bowel obstruction; transitional zone was detected in twenty-three patients.

\section{Bowel loop telescoping}

This was encountered in only one intussusception patient (2\%) who had positive lead point neoplastic mass lesion.

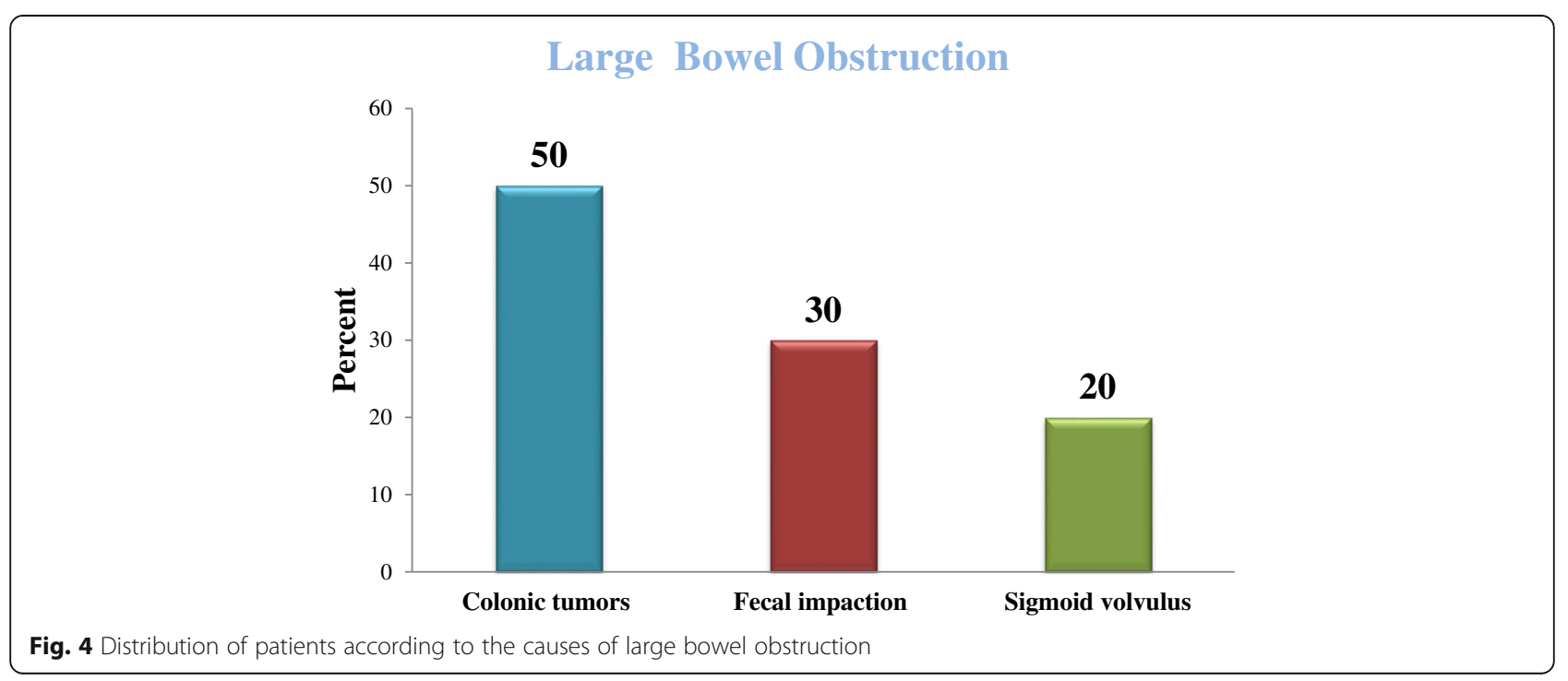




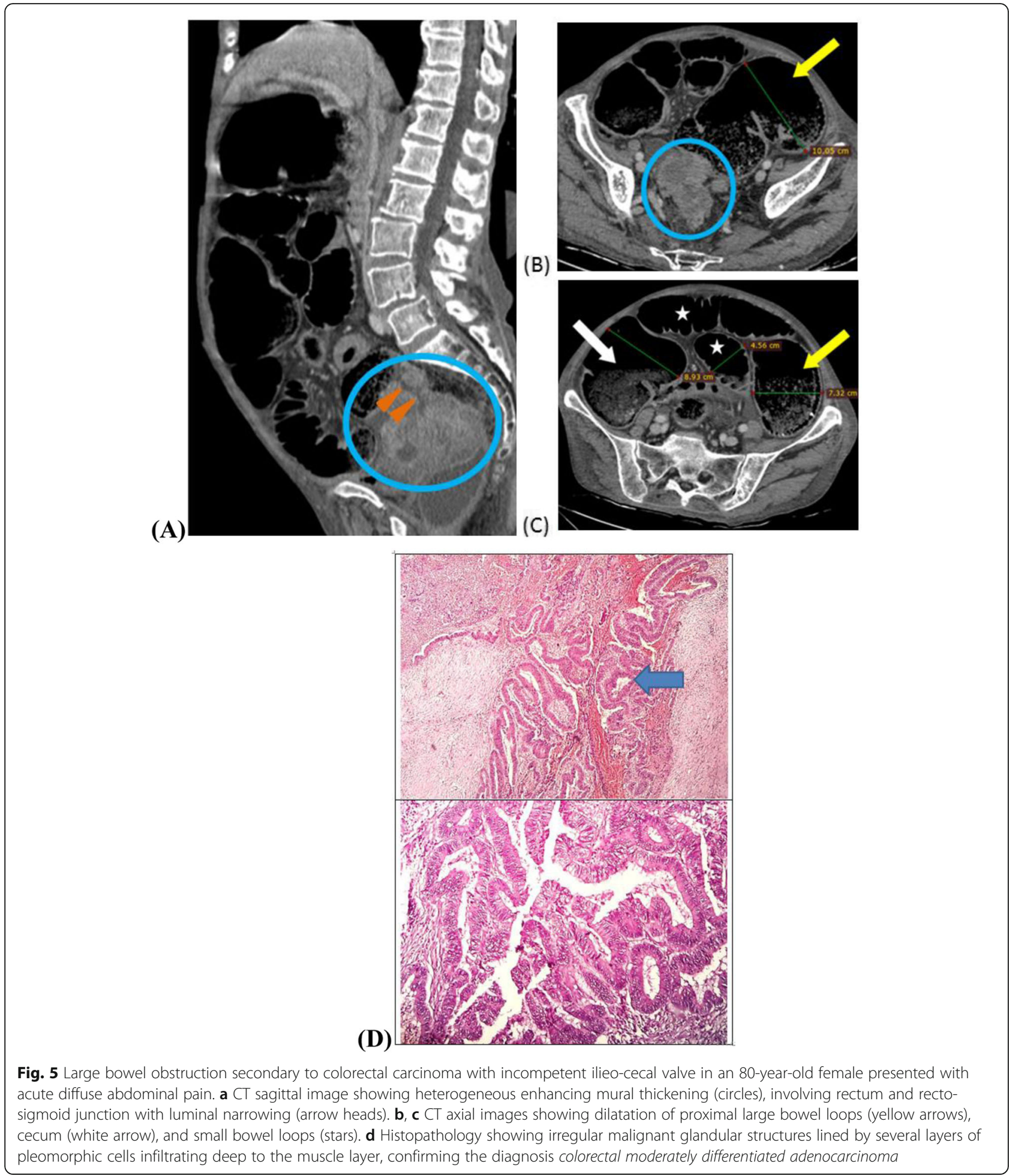

\section{Mesenteric vessel abnormalities}

Mesenteric vessel abnormalities were detected in twenty patients. More than one sign can be detected in the same patient. Twelve patients $(60 \%)$ had engorged mesenteric vessels, and one out of the twelve showed a comb sign in active Crohn's disease. Four patients (20\%) had thrombosed mesenteric vessels in MVO conditions. Four patients $(20 \%)$ had twisted mesenteric vessels, giving a whirl sign, seen in sigmoid volvulus, closed loop small bowel obstruction and mesenteric malrotation. Three patients (15\%) had mesenteric venous gas in MVO conditions. One patient (5\%) had invaginated 


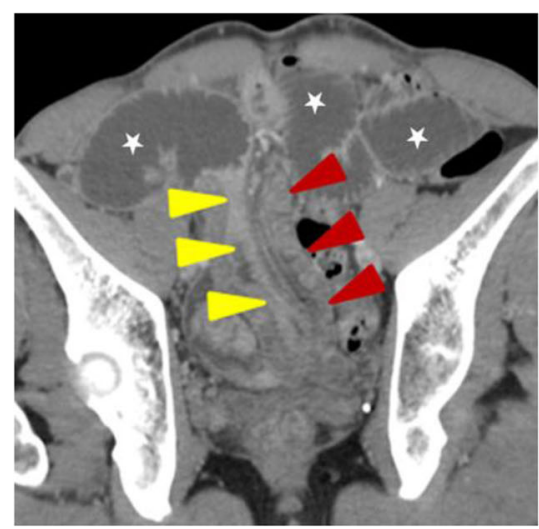

(A)

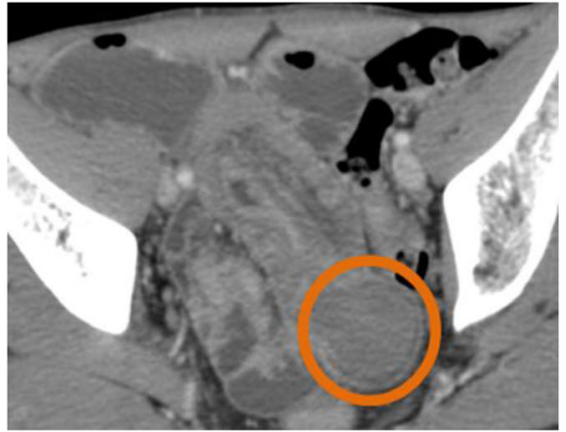

(C)

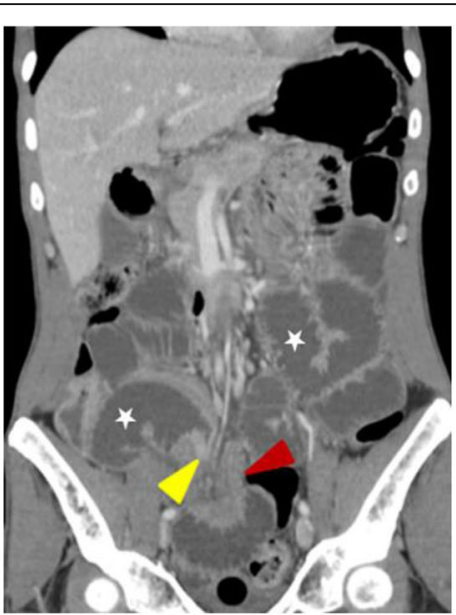

(B)

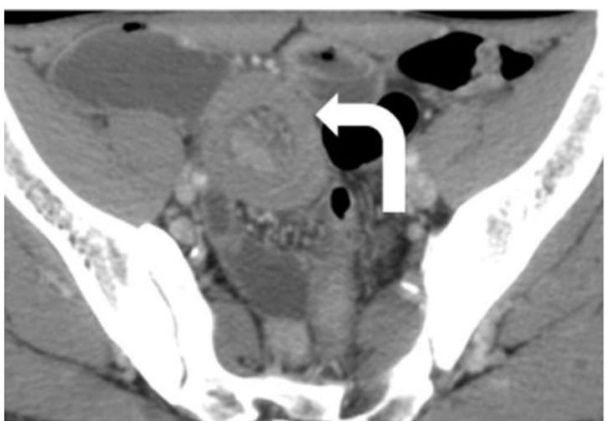

(D)

Fig. 6 Entero-enteric intussusception in a 65-year-old male presented with acute abdominal pain and vomiting. Axial CT image (a) and coronal CT image (b) showing invagination of distal jejunal bowel loops (the intussuceptum) (yellow arrowheads) into proximal ileal bowel loops (the intussuscipient) (red arrowheads) with subsequent dilated proximal small bowel loops (stars). c Axial CT image showing the lead point for this intussusception; intra-mural soft tissue mass lesion (circle). Sausage shape appearance is seen in axial CT images (a \& c). $\mathbf{d}$ Axial CT image at another level showing the typical target sign (curved arrow)

mesenteric vessels during bowel loop telescoping in intussusception.

Mesenteric fat changes were found in twenty patients $(40 \%)$ in the form of mesenteric fat stranding due to different pathological causes in acute setting. Intraperitoneal fluid collection was detected in thirty-seven patients (74\%): free $(73 \%)$ and loculated (27\%), pneumoperitoneum was detected in seven patients $(14 \%)$ in perforated conditions, and pneumatosis intestinalis was seen in three patients $(6 \%)$ in acute mesenteric ischemia.

\section{The final diagnosis}

The final diagnosis was confirmed by the following:

- Surgical findings in 29 patients underwent surgical intervention.

- Surgical findings and histopathological examination were done in 13 patients.
- In 8 patients, where surgery and histopathological examination were not indicated, final diagnosis was confirmed by clinical response to medical treatment.

\section{Assessment of the role of MDCT in the evaluation of gastrointestinal tract-related acute abdominal pain in geriatric patients}

The role of multidetector computed tomography (MDCT) in evaluating gastrointestinal tract-related acute abdominal pain in geriatric patients was very high as its sensitivity (95\% CI) was $98(89.35 \%$ to $99.95 \%)$ as its PPV (95\%CI) was 100 (92.75\% to $100 \%)$.

\section{Discussion}

Evaluation of the elderly patient with abdominal pain can be difficult, time-consuming, and fraught with potential missteps [2]. As the population continues to age, the utilization of CT imaging in elderly patients presenting to the ED becomes increasingly important [9]. CT 


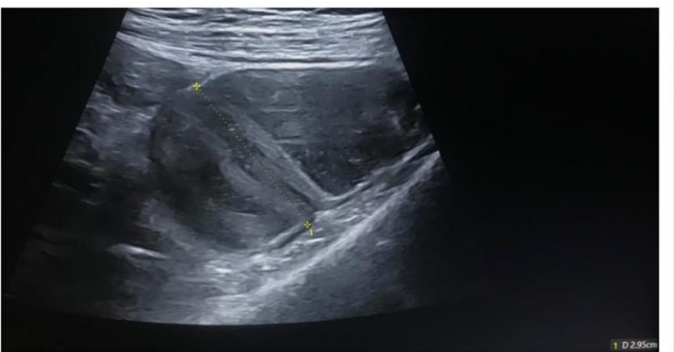

(A)

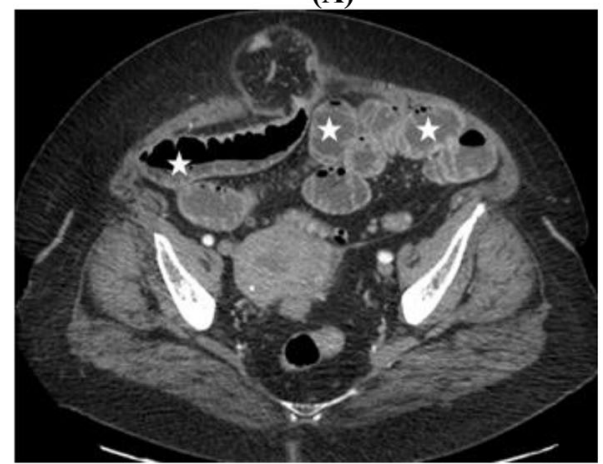

(C)

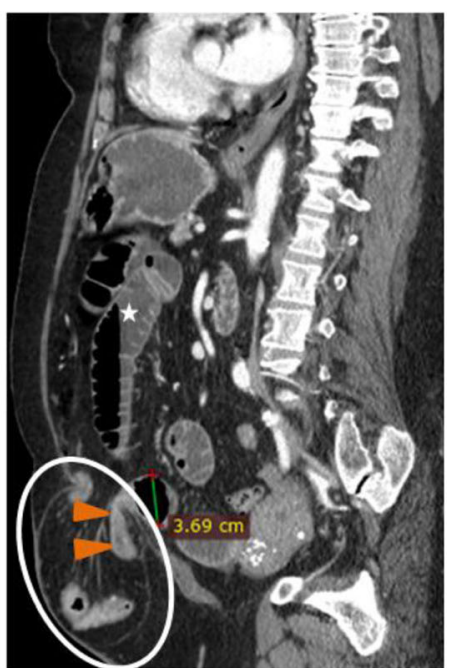

(B)

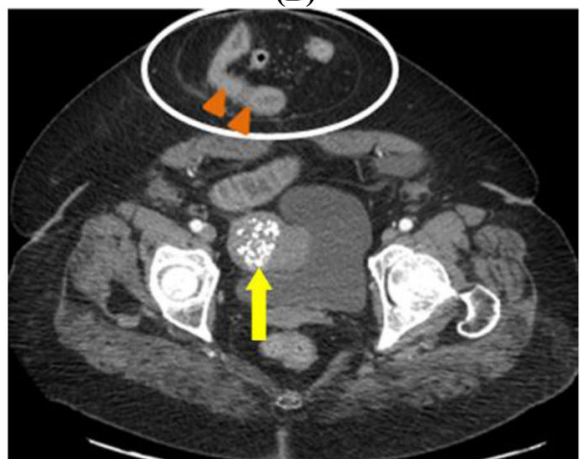

(D)

Fig. 7 High-grade mechanical small bowel obstruction caused by paraumbilical hernia in a 78-year-old female patient complaining of acute abdominal pain. a Abdominal ultrasound image showing dilated small bowel loops. CT sagittal image (b) and CT axial images (c, d), showing an hernial sac (circle) containing collapsed mid-ileal bowel loops (arrowheads) with consequent dilatation of the ileal and jejunal bowel loops proximal to herniated loop (stars). A calcified uterine fibroid (yellow arrow) is incidentally seen on (d) axial CT image

provides a global perspective of the gut, mesenteries, omenta, peritoneum, and retroperitoneum, uninhibited by the presence of bowel gas and fat [10].

Obtaining a careful medical history and performing a clinical examination are the initial diagnostic steps for patients with acute abdominal pain. On the basis of the results of this clinical evaluation and laboratory investigations, the clinician considered imaging examinations to help establish the correct diagnosis. Findings after using erect abdominal X-ray and abdominal ultrasound were inconclusive, and CT was mandatory for diagnosing the etiology of gastrointestinal pain in the studied cases.

In the current study, acute abdominal pain due to intestinal causes in 42 patients (84\%) was more common than due to gastric causes in 8 patients (16\%). This is matching with Gardner et al. [9] among their study on elderly patients with acute abdominal pain which found that intestinal causes (SBO, diverticulitis, appendicitis, bowel ischemia, and colonic obstruction) were the predominant causes of acute abdominal pain.
In the current study, regarding gastric causes of acute abdominal pain in geriatric patients, gastric obstruction was noted in 8 patients $(62.5 \%)$, while gastric perforation was seen in 3 patients (37.5\%); perforated PUD was the main cause of gastric perforation followed by gastric carcinoma. This is in agreement with Hainaux et al. [11] who reported that the perforation of gasteroduodenal ulcer is much common than perforated gastric carcinoma. Chang et al. [3] reported that the incidence of peptic ulcer disease is increasing in the elderly owing in part to the increasing availability and use of aspirin and non-steroidal anti-inflammatory drugs (NSAIDs) with high risk of developing complications as perforation.

Based on MDCT findings, intestinal obstruction was the most common intestinal etiology of acute abdominal pain among these geriatric patients which represented $71.4 \%$ of intestinal causes followed by acute inflammatory conditions (28.6\%), intestinal perforation (23.8\%), and acute vascular etiologies (11.9\%). Only one patient had colo-cholecystic fistula $(2.4 \%)$. This is matching with 


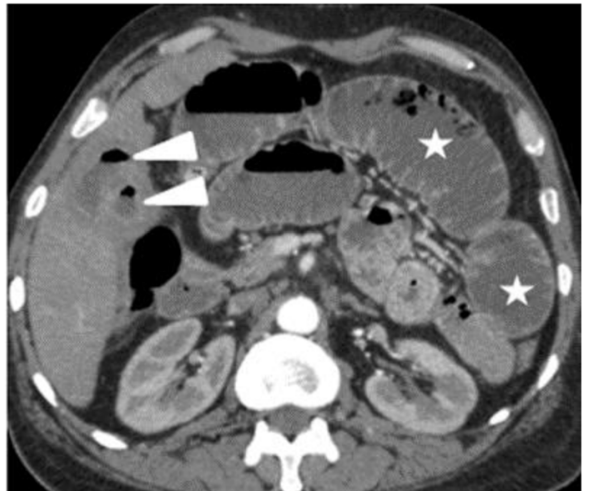

(A)

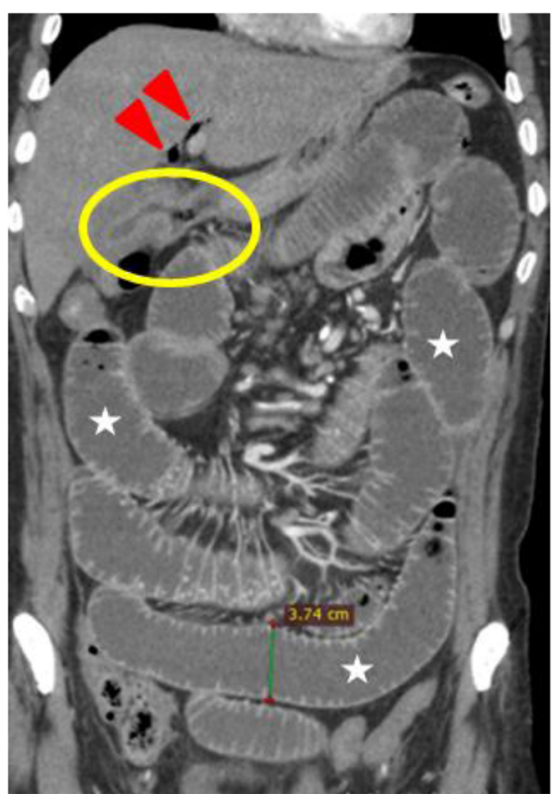

(B)

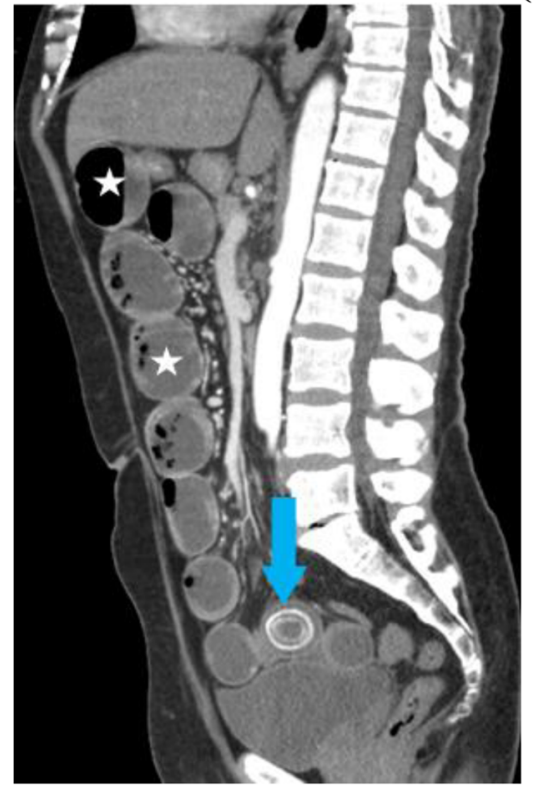

(C)

Fig. 8 Gall stone ileus in a 67-year-old male patient presented with acute abdominal pain, fever, and vomiting. Post IV contrast CT abdomen and pelvis (a) axial and (b) coronal and (c) sagittal images showing fistula (circle) between the gall bladder and duodenum with multiple intraluminal air fluid levels seen in the gall bladder (white arrowheads), minimal pneumobilia (red arrowheads) and lamellated impacted stone seen at the proximal ileal bowel loop (arrow) with distended proximal small bowel loops (stars)

the study done by Pinto et al. [12] among the elderly patients presented with acute abdominal pain that found bowel obstruction was the commonest etiology followed by inflammatory, perforation, and vascular etiologies.

In the present study, small bowel obstruction more commonly occurred $(66.7 \%)$ with proximally dilated small bowel loops than large bowel obstruction (33.3\%) with proximally dilated large bowel loops. This is in agreement with Gardner et al. [9] and Hendrickson et al.'s [13] studies among elderly patients that small bowel obstruction was more common than large bowel obstruction, and Jackson et al. [14] noted that in patients with intestinal obstruction, the bowel loops are seen dilated proximal to the site of obstruction and decompressed distally.

In the current study, external hernias were the most common causes of small bowel obstruction. This is different 


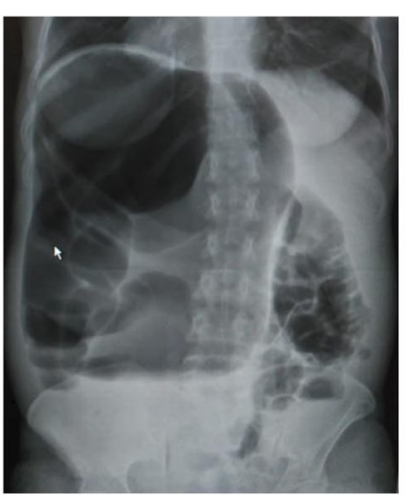

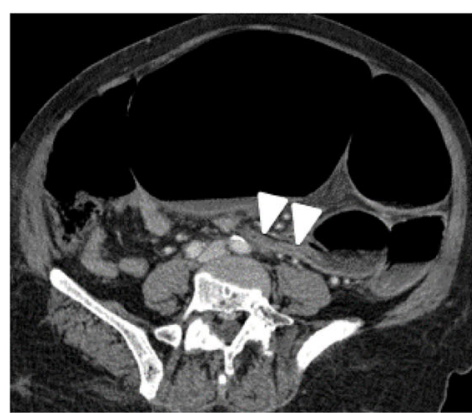

(B)

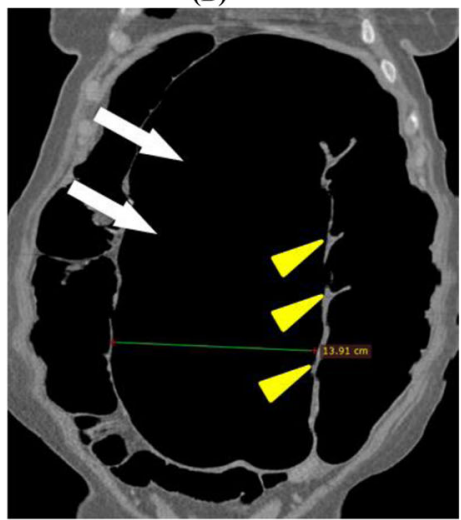

(D)

(A)

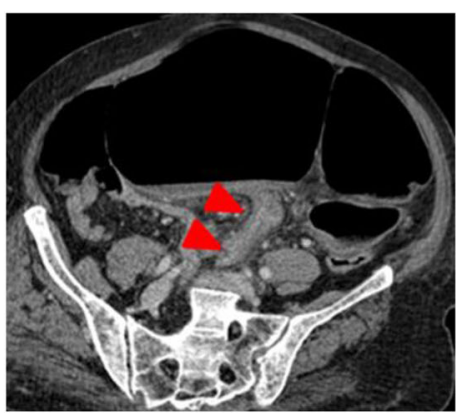

(C)

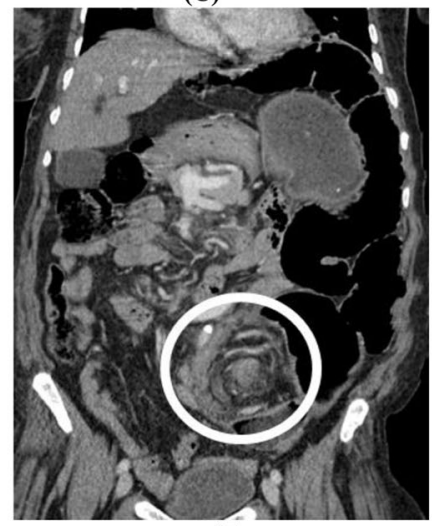

(E)

Fig. 9 Sigmoid volvulus in a 75-year-old female patient presented with acute abdominal pain and distension. a Plain erect abdominal film showing distended sigmoid loop with inverted $U$ configuration and coffee bean sign. b Post contrast axial CT image showing a proximalsuperior sigmoid transition (white arrowheads). c Post contrast axial CT image showing distal-inferior sigmoid transition (red arrowheads), which is oriented in the direction opposite to the proximal transition. $\mathbf{d}$ Coronal CT reformatted image showing markedly dilated gas- and feces-filled sigmoid loop (white arrows) with loss of its haustral markings (yellow arrowheads) and extended from the pelvis way up to the left upper abdomen, giving an inverted $U$ configuration, forming a closed-loop obstruction. e Coronal $C T$ image showing twisting of the mesentery and mesenteric vessels of sigmoid colon, giving a whirl sign (circle)

from the study of Henry et al. [15] among the elderly patients that found the main causes for small intestinal obstruction were adhesions followed by hernia. Meanwhile, Hendrickson et al. [13] reported that hernias are extremely important to recognize in the elderly because this group has a high rate of strangulation and bowel infarction. This difference might be explained as hernias are common among the geriatric population because of loss of strength of the abdominal wall which was seen in 5 cases of the current study as well as majority of the studied patients with small bowel obstruction did not undergo previous abdominal operations; therefore, they did not have a risk for obstruction due to postoperative adhesions; only two patients had adhesions due to prior abdominal surgeries with consequent small bowel obstruction.

In the present study, primary colonic tumors were the most common causes of large bowel obstruction, 5 out of 10 cases of large bowel obstruction (LBO) were due to colonic tumors followed by fecal impaction in 3 cases and sigmoid volvulus in 2 cases. This is matching with 


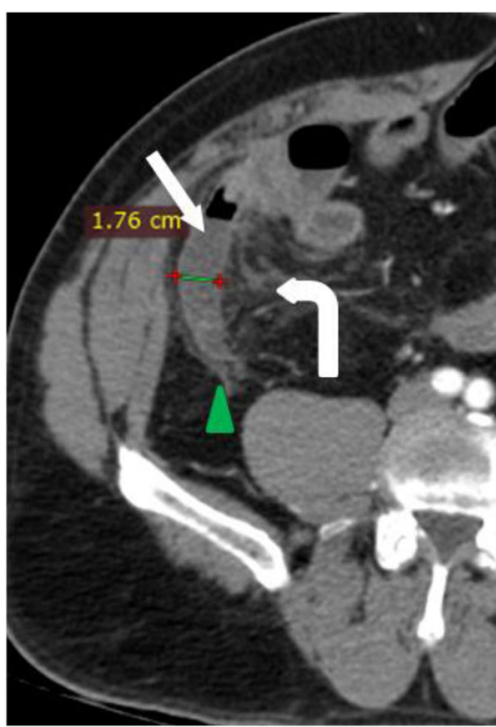

(A)

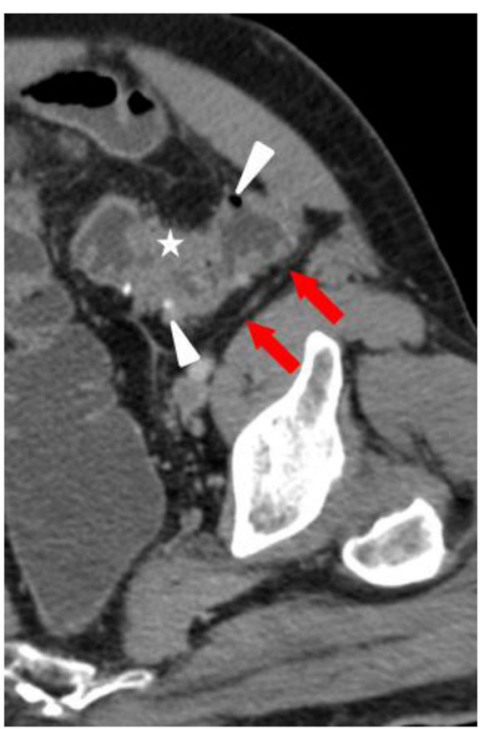

(B)

Fig. 10 Acute appendicitis and acute divertculitis in a 68-year-old male patient presented with acute lower abdominal pain. a, b Focused axial CT images following water enema and intravenous contrast injection. a Showing dilated appendix (white arrow) with related extensive fat stranding (curved arrow) and minimal free fluid collection (green arrowhead). b Showing sigmoid wall thickening (star) and diverticular outpouchings (white arrowheads) with related mild fat stranding (red arrows)

Scott et al. [1] and Lyon et al. [16] who reported that primary colonic tumor was the most common etiology of LBO in the elderly. On CT basis, tumors showed asymmetrical focal heterogeneous enhancement. This is relatively coping with Sheikh et al.'s [17] study among bowel diseases that found marked asymmetrical wall thickening and focal bowel wall involvement with heterogeneous enhancement in neoplastic diseases.

In the current study, mechanical bowel obstruction $(86.7 \%)$ was due to various causes as external hernias, colonic tumors, gall stone ileus, fecal impaction, sigmoid volvulus, postoperative adhesions, intussusception, active

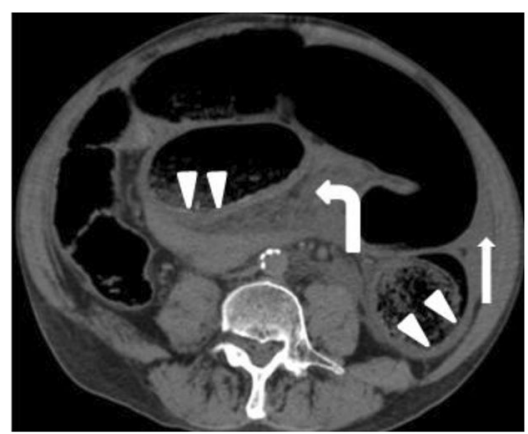

(A)

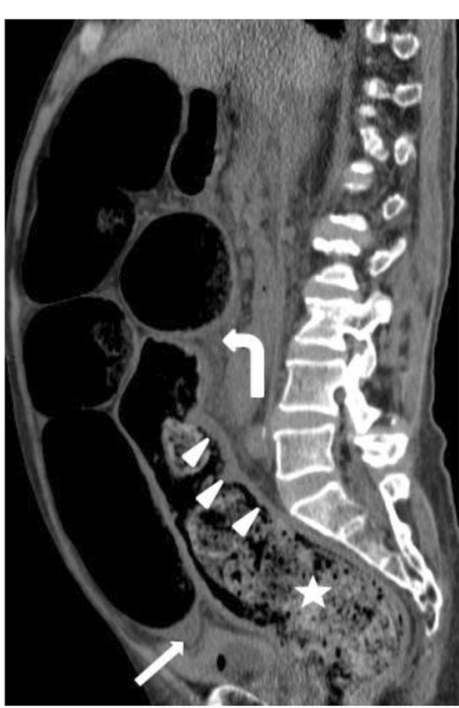

(B)

Fig. 11 Fecal impaction and sterocoral colitis in a 65-year-old man presented with constipation and acute abdominal pain. CT abdomen and pelvis non-contrast phase (a) axial image and (b) sagittal image, showing a large volume of fecal matter distending the rectum, sigmoid colon, and transverse colon (star), colon wall thickening with sub mucosal edema (white arrowheads), extensive pericolonic fat stranding (curved arrow) and free fluid collection (arrow) 
Table 4 Distribution of patients according to intestinal inflammatory causes as regards occurrence of complications

\begin{tabular}{|c|c|c|c|c|}
\hline \multirow[t]{3}{*}{ Intestinal inflammatory causes } & \multicolumn{4}{|c|}{ Complications } \\
\hline & \multicolumn{2}{|c|}{ Negative } & \multicolumn{2}{|c|}{ Positive } \\
\hline & No & $\%$ & No & $\%$ \\
\hline Acute diverticulitis & 1 & 8.3 & 4 & 33.3 \\
\hline Acute appendicitis & 1 & 8.3 & 3 & 25 \\
\hline Acute diverticulitis \& acute appendicitis & 1 & 8.3 & 0 & 0 \\
\hline Active Crohn's disease & 0 & 0 & 1 & 8.3 \\
\hline Stercoral colitis & 1 & 8.3 & 0 & 0 \\
\hline Total & 4 & 33.3 & 8 & 66.7 \\
\hline
\end{tabular}

Crohn's disease, phytobezoar, and bowel hematoma; 4 cases were diagnosed by MDCT as gall stone ileus. Similarly, Morano et al. [18] and Scott et al. [1] reported that gallstone ileus, which is rare in the general population, is more common in the elderly and can lead to short bowel obstruction (SBO). Maddu et al. [19] reported that incidence of fecal impaction is increased with age particularly in institutionalized elderly patients and constituted $12 \%$ of intestinal obstruction in Henry et al.'s [15] study among elderly patients.

In the present study, inflammatory etiology represented $28.6 \%$ of intestinal causes of acute abdominal pain among the studied patients; most of them were acute diverticulitis and acute appendicitis with subsequent complications which occurred in $66.7 \%$ of inflammatory conditions. This is more or less similar to the study done on the elderly patients by Pinto et al. [12] that found intestinal inflammatory causes of acute abdominal pain to represent $21.8 \%$; Chang et al. [3] and Storm-Dickerson [20] both stated that complications of acute diverticulitis and acute appendicitis are more frequent in the elderly.

In the current study, intestinal perforation due to inflammatory causes was more common than due to neoplastic causes. Launay-Savary et al. [21] reported that colonic perforation in the elderly is most often secondary to complications of diverticular disease, while colonic perforations secondary to cancer are rare. Detection of

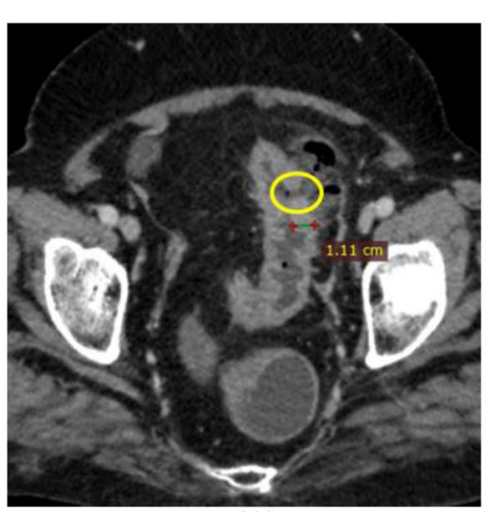

(A)

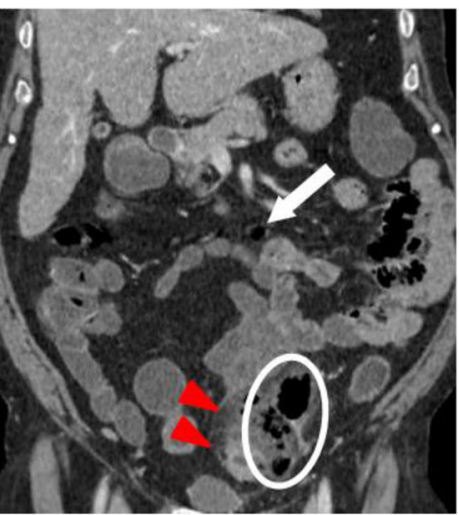

(B)

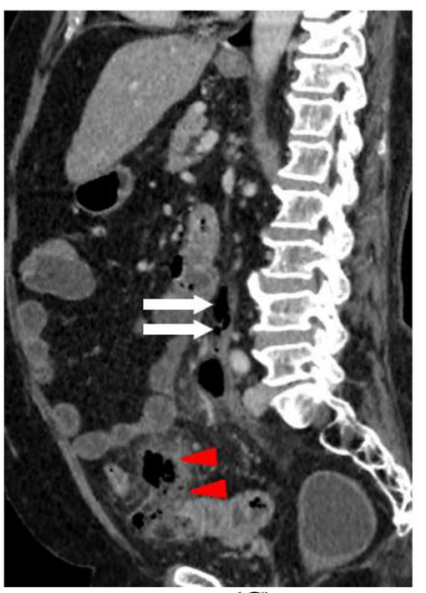

(C)

Fig. 12 Acute perforated diverticulitis in a 67-year-old female patient presented with acute abdominal pain. CT abdomen and pelvis images following water enema and intravenous contrast injection. a Axial image showing sigmoid wall thickening with focal mural defect (yellow circle). b Coronal and c sagittal images, showing gas-containing abscess (white circle), extra luminal air (arrows), and related extensive fat standing (arrowheads) 


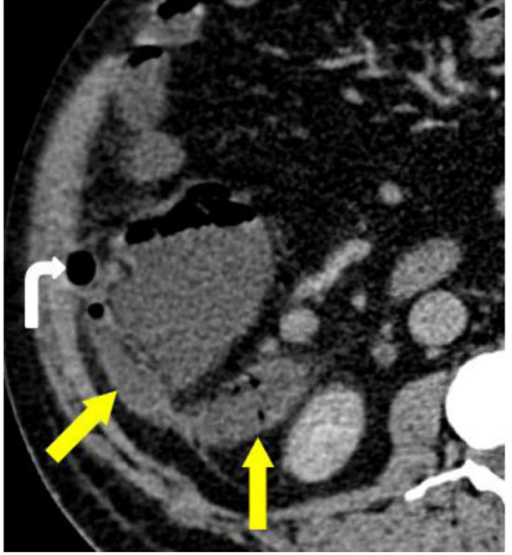

(A)

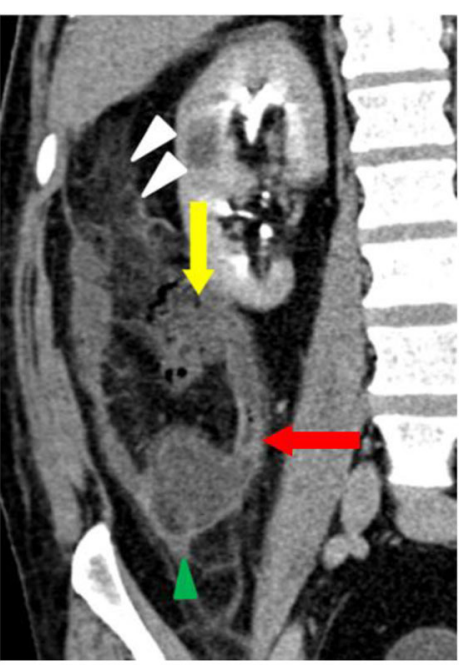

(B)

Fig. 13 Acute perforated appendicitis with abscess formation in a 66-year-old male patient presented with acute right iliac fossa pain, vomiting, and fever. Focused CT axial (a) and focused coronal (b) images following water enema and intravenous contrast injection CT abdomen and pelvis, showing inflamed appendix (red arrow) with its tip is seen continuous with an abscess containing gas bubbles (yellow arrows), free gas is seen at the retrocecal region (curved arrow) with related mild fat stranding (white arrowheads) and minimal free fluid collection (green arrowheads)

pneumoperitoneum is of paramount importance for the timely diagnosis of gastrointestinal tract perforation. In the present study, pneumoperitoneum was detected in 7 patients (14\%), and 2 out of 7 showed leakage of the oral contrast material on MDCT, surgically proving gastrointestinal tract perforation. This is typically matching with Maniatis et al.'s [22] report that extraluminal air or extravasation of the oral contrast medium is considered to be the direct sign of GI perforation.

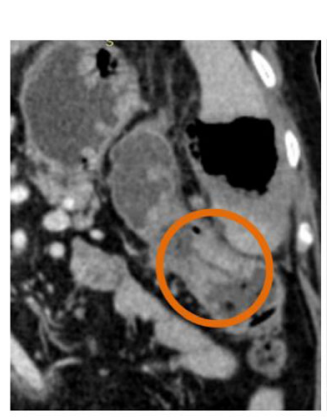

(A)

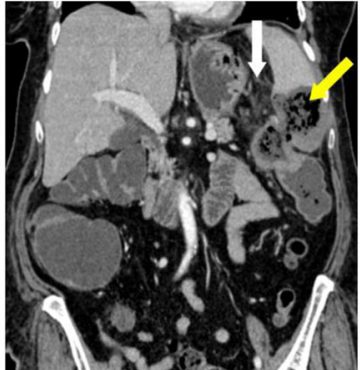

(B)

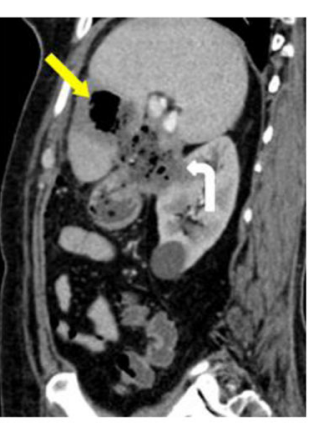

(C)

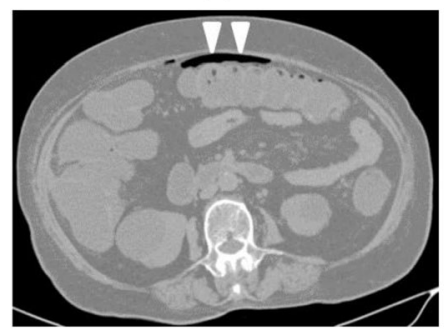

(D)

Fig. 14 Perforated colonic carcinoma in a 70-year-old female patient presented with acute left hypochondrial pain and fever. a Focused coronal CT image showing asymmetric circumferential focal mural thickening involving splenic flexure and proximal descending colon with luminal narrowing "shouldering sign" (circle). b Coronal CT and c sagittal reformatted CT images showing infiltration of lower part of spleen with localized collection containing gas and fecal matter within the splenic substance, surrounded by hypodense splenic matrix (yellow arrow) with adjacent mild fat stranding (white arrow) and infiltration of the anterior aspect of the left renal parenchyma (curved arrow). $\mathbf{d}$ Axial CT (lung window) image showing free pneumoperitoneum (arrowhead), indicating perforation 


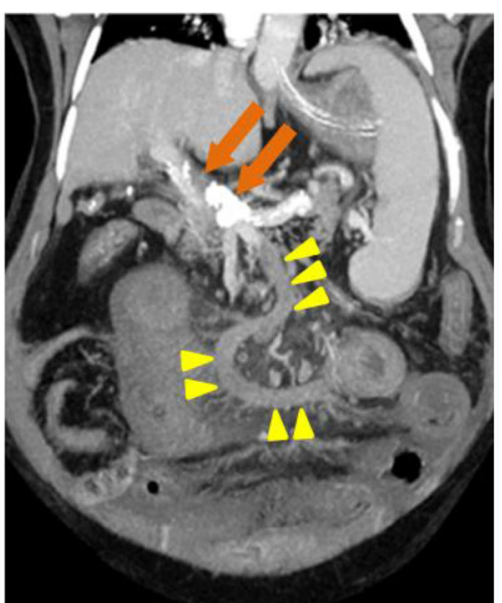

(A)

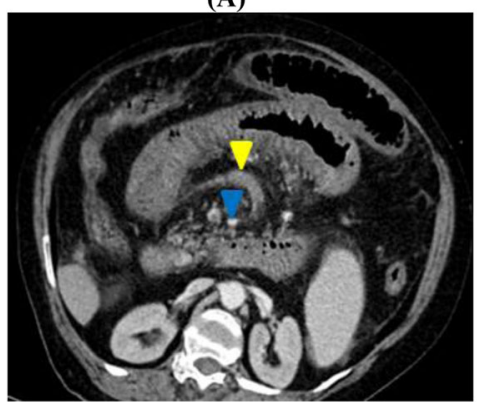

(C)

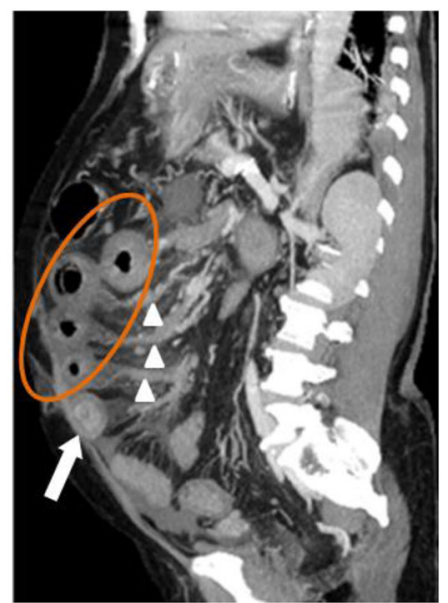

(B)

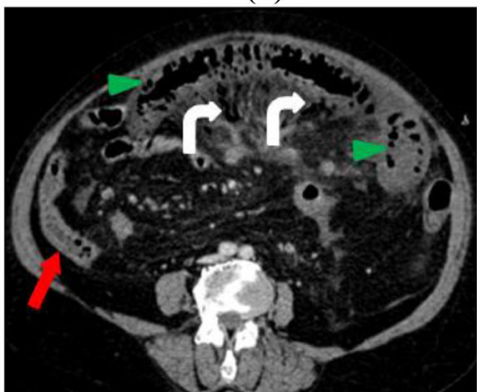

(D)

Fig. 15 A 65-year-old female patient presented with acute abdominal pain. a Coronal, b sagittal maximum intensity projection (MIP) CT images, showing partially thrombosed portal vein yet with good contrast opacification with old calcified thrombus seen within (orange arrows) with total thrombosis of the SMV (yellow arrowheads) (which has malrotation configuration with SMA (blue arrowhead) seen on (c) axial CT image) as well as its visualized distal branches (white arrowheads) and submucosal edema with non-enhancing affected bowel loops (circle) and hypoenhancing bowel loops (white arrow), giving a target sign. $\mathbf{d}$ Axial CT image showing non-enhancing distended bowel loops with diffuse pneumatosis intestinalis (green arrow heads), compared with normal enhancing ileal bowel loop (red arrow) with mesenteric venous gas is seen (curved arrows)

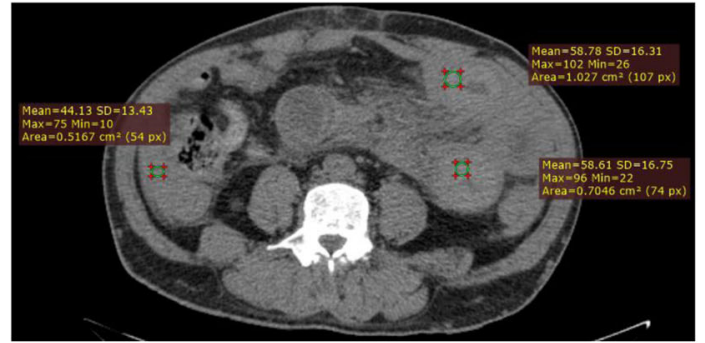

(A)

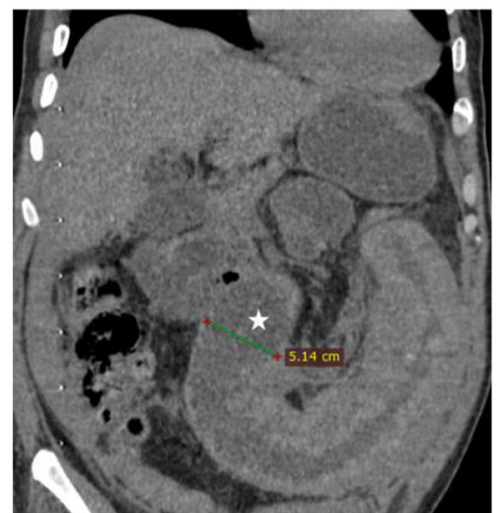

(B)

Fig. $16 \mathrm{CT}$ of abdomen and pelvis in non-contrast phase. a Axial image and $\mathbf{b}$ coronal reformatted image in a 72-year-old male patient with given history of warfarin intake, diagnosed with intra-mural small bowel hematoma, showing hyperdense mural thickening with attenuation density of $58 \mathrm{HU}$ on non-contrast CT phase and free intraperitoneal fluid collection of high attenuation (44 HU) with consequent proximal small bowel dilatation (star) 


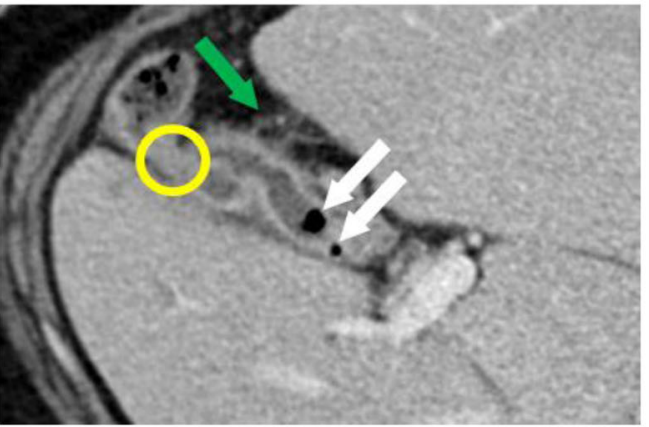

(A)

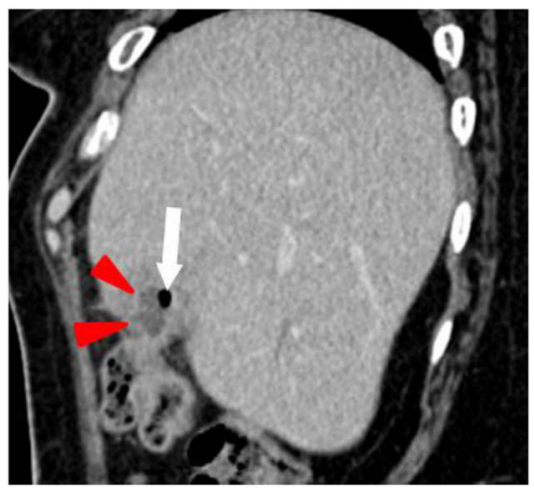

(B)

Fig. 17 Colo-cholecystic fistula with subsequent cholecystitis in a 65-year-old female patient presented with right hypochondrial pain and fever with history of gall bladder biopsy. Post contrast axial CT reformatted image (a) and sagittal reformatted image (b) showing irregular outline of gall bladder (red arrowheads) with multiple intra-luminal air foci (white arrows), its fundus tethered to hepatic flexure (yellow circle) with related mild fat stranding (green arrow)

Acute intestinal ischemia was seen as vascular etiology of acute abdominal pain among the studied elderly patients; end-stage irreversible ischemic insults were seen in 3 patients (6\%) with occlusive arterial, and venous changes showed pneumatosis intestinalis and coupled with mural non-enhancement correlated with final surgical findings. Only one patient showing reversible bowel ischemia as a sequel to SMV occlusive changes showed diminished bowel enhancement. This is matched with Treyaud et al.'s [23] findings that stated pneumatosis intestinalis as commonly seen in irreversible ischemic mural changes. Also, presence of portal venous gas is highly diagnostic to ischemic changes, and this in parallel to Millet et al. [5] who indicate that bowel ischemia was uncommon in elderly patients as an etiology of acute abdominal pain.

One of the main advantages of contrast-based CT studies is assessment of the supplying vessel abnormalities. Patients with suspected vascular insults gain the main benefit. This is matching with the study of Florim et al. [24] which reported that acute arterial thrombi and emboli may appear as obvious low-attenuation filling defects on contrast-enhanced CT in the SMA lumen and reported that low-attenuation filling defect on contrast-enhanced CT of the superior mesenteric vein has higher specificity values in venous acute mesenteric ischemia. Florim et al. [24] also reported portomesenteric venous gas as less common but more specific finding of acute bowel ischemia, being present in $3-14 \%$ of cases.

Spontaneous intramural bowel hematoma was detected in one elderly patient in the current study with excessive anticoagulant intake, presented with acute abdominal pain. Samie et al. [25] stated the incidence of spontaneous intramural small bowel hematoma secondary to anticoagulant therapy is predicted to further increase as a result of the wide use of long-term anticoagulation in an aging population.

Summation of the current MDCT findings was correlated with the final diagnosis, whether confirmed by surgical intervention, histopathological examination, or good response to medical treatment. There was only one misdiagnosis; a hypothesized acute diverticulitis and associated colonic wall thickening was highly suspicious for cancer. In this case, the patient underwent further colonoscopy examination, and the following histology excluded cancer. So MDCT has a very high sensitivity of $98 \%$ and positive predictive value of $100 \%$ in diagnosing the causative pathological condition of acute gastrointestinal pain in these geriatric patients. This is in parallel to Reginelli et al. [6] who stated that the usefulness of CT in the diagnosis and management of acute gastrointestinal emergencies is well established and confirmed by the results of their study, in which the CT diagnosis correlated with the final diagnosis in very high rate, and Chang et al. [3] reported that with its proven ability to diagnose a wide variety of conditions, CT remains the diagnostic modality of choice for imaging the acute abdomen in the elderly.

\section{Limitations}

Some limitations that were encountered in the current study included the wide varieties of pathologies that cause gastrointestinal-related acute abdominal pain like obstructive, inflammatory, perforation, ischemic, and others lesions. This variation affected the sample size in each pathological category. Further studies on a large number of patients may be of value in retrieving better results with more understanding of the gastrointestinal pathologies causing abdominal pain. 


\section{Conclusion}

MDCT is a reliable diagnostic imaging modality for geriatric patients presented with acute abdominal pain suggested to be of gastrointestinal origin with a very high sensitivity in diagnosing the causative pathological conditions. MDCT efficiently differentiates between obstructive, inflammatory, perforated, and ischemic bowel disorders and so reaching the accurate diagnosis with consequent proper prognosis.

\begin{abstract}
Abbreviations
ED: Emergency department; MDCT: Multidetector computed tomography; GI: Gastrointestinal; SPSS: Statistical Package for the Social Sciences;

SMA: Superior mesenteric artery; SMV: Superior mesenteric vein; MVO: Mesenteric vascular occlusion; PUD: Peptic ulcer disease; SBO: Small bowel obstruction; LBO: Large bowel obstruction; MIP: Maximum intensity projection; kVp: Kilovoltage peak; mAs: Milliampere-seconds; NSAIDs: Nonsteroidal anti-inflammatory drugs
\end{abstract}

\section{Acknowledgements}

Not applicable

\section{Authors' contributions}

DE did the writing of the draft and revision of the final manuscript. AR did the revision of the final manuscript. AA did the writing of the draft. AE did the editing of the draft and revision of the final manuscript. All authors have read and approved the manuscript.

\section{Funding}

Not applicable

\section{Availability of data and materials}

The datasets used and/or analyzed during the current study are available from the corresponding author on reasonable request.

\section{Ethics approval and consent to participate}

Approval for this prospective study was obtained from the Research Ethics Committee of Alexandria Faculty of Medicine (Ethics committee's reference number: 0106128, IRB No.: 00012098, FWA No.: 00018699). All study procedures were carried out in accordance with the Declaration of Helsinki regarding research involving human subjects. Written informed consent was obtained from the patients.

\section{Consent for publication}

Consent for the use of patients' data and medical record was obtained.

\section{Competing interests}

The authors declare that they have no competing interests.

Received: 10 December 2020 Accepted: 24 January 2021

Published online: 22 February 2021

\section{References}

1. Scott J, Holder J, Cort K (2017) Acute abdominal pain in the elderly. Contemp Diagn Radiol 40(9):1-8

2. Leuthauser A, McVane B (2016) Abdominal pain in the geriatric patient. Emerg Med Clin North Am 34(2):363-375

3. Chang CC, Wang SS (2007) Acute abdominal pain in the elderly. Int J Gerontol 1(2):77-82

4. Spangler R, Van Pham T, Khoujah D et al (2014) Abdominal emergencies in the geriatric patient. Int J Emerg Med 7(1):43

5. Millet I, Alili C, Bouic-Pages E et al (2013) Journal club: acute abdominal pain in elderly patients: effect of radiologist awareness of clinicobiologic information on CT accuracy. AJR Am J Roentgenol 201(6):1171-1179

6. Reginelli A, Russo A, Pinto A et al (2014) The role of computed tomography in the preoperative assessment of gastrointestinal causes of acute abdomen in elderly patients. Int J Surg 12(Suppl -2):S181-S186

7. Leschka S, Alkadhi H, Wildermuth S et al (2005) Multi-detector computed tomography of acute abdomen. Eur Radiol 15(12):2435-2447
8. Cahir JG, Freeman AH, Courtney HM (2004) Multislice CT of the abdomen. Br J Radiol 77(Suppl-1):S64-S73

9. Gardner CS, Jaffe TA, Nelson RC (2015) Impact of CT in elderly patients presenting to the emergency department with acute abdominal pain. Abdom Imaging 40(7):2877-2882

10. Hill BC, Johnson SC, Owens EK et al (2010) CT scan for suspected acute abdominal process: impact of combinations of IV, oral, and rectal contrast. World J Surg 34(4):699-703

11. Hainaux B, Agneessens E, Bertinotti R et al (2006) Accuracy of MDCT in predicting site of gastrointestinal tract perforation. AJR Am J Roentgenol. 187(5):1179-1183

12. Pinto A, Sparano A, Gagliardi N et al (2010) Acute abdominal pain in the elderly patient: impact of early MDCT examination on diagnosis and management. ECR 2010(C-1464):1-9. https://doi.org/10.1594/ecr2010/C-1464

13. Hendrickson M, Naparst TR (2003) Abdominal surgical emergencies in the elderly. Emerg Med Clin North Am 21(4):937-969

14. Jackson PG, Raiji M (2011) Evaluation and management of intestinal obstruction. Am Fam Physician 83(2):159-165

15. Henry MA, Lerco MM, Oliveira WK et al (2007) Intestinal obstruction in the elderly. ABCD. Arq Bras Cir Dig 20(4):225-229

16. Lyon C, Clark DC (2006) Diagnosis of acute abdominal pain in older patients. Am Fam Physician 74(9):1537-1544

17. Sheikh MT, Sheikh MT, Jan M et al (2017) Role of multi-detector CT (MDCT) in evaluation of bowel diseases. J Clin Diagn Res 11(7):TC11-TC13

18. Morano WF, Bowne WB (2020) Small bowel obstruction in the elderly. In: Rosenthal RA, Zenilman ME, Katlic MR (eds) Principles and practice of geriatric surgery. Springer, Switzerland, pp 991-1011. https://doi.org/10. 1007/978-3-319-47771-8 64

19. Maddu KK, Mittal P, Arepalli CD et al (2014) Colorectal emergencies and related complications: a comprehensive imaging review-noninfectious and noninflammatory emergencies of colon. AJR Am J Roentgenol 203(6): 1217-1229

20. Storm-Dickerson TL, Horattas MC (2003) What have we learned over the past 20 years about appendicitis in the elderly? Am J Surg 185(3):198-201

21. Launay-Savary MV, Rainfray M, Dubuisson V (2015) Emergency gastrointestinal surgery in the elderly. J Visc Surg 152(6):S73-S79

22. Maniatis V, Chryssikopoulos H, Roussakis A et al (2000) Perforation of the alimentary tract: evaluation with computed tomography. Abdom Imaging 25(4):373-379

23. Treyaud MO, Duran R, Zins M et al (2017) Clinical significance of pneumatosis intestinalis - correlation of MDCT-findings with treatment and outcome. Eur Radiol 27(1):70-79

24. Florim S, Almeida A, Rocha D et al (2018) Acute mesenteric ischaemia: a pictorial review. Insights Imaging 9(5):673-682

25. Samie AA, Sun R, Huber A et al (2013) Spontaneous intramural small-bowel hematoma secondary to anticoagulant therapy: a case series. Med Klin Intensivmed Notfmed 108(2):144-148

\section{Publisher's Note}

Springer Nature remains neutral with regard to jurisdictional claims in published maps and institutional affiliations.

\section{Submit your manuscript to a SpringerOpen ${ }^{\circ}$ journal and benefit from:}

- Convenient online submission

- Rigorous peer review

- Open access: articles freely available online

High visibility within the field

- Retaining the copyright to your article

Submit your next manuscript at $>$ springeropen.com 